\title{
Nuevo papel de la didáctica implementada en las aulas universitarias
}

\author{
Zunilda Ramona Paredes de Ríos \\ zunildap@hotmail.es
}

\section{Dominga Elsa Velázquez}

domingaelsa@gmail.com

Universidad Nacional de Pilar

\section{RESUMEN}

Esta investigación tiene el propósito de describir el papel de la didáctica implementada en las aulas universitarias, y a su vez destacar la relevancia del sujeto como núcleo central de este proceso. El método utilizado es la revisión y análisis exhaustivos de la literatura existente con relación al tema abordado. Los hallazgos principales de este trabajo fueron: que el papel de la didáctica implementada en las aulas universitarias requiere la articulación de procesos cognitivos para el logro del desarrollo de competencias y capacidades a través de experiencias de aulas sistemáticas y racionales, utilizando diversas herramientas tecnológicas para el acceso a la información y para transferirla de manera significativa a los estudiantes, tanto en clases presenciales como virtuales, en situaciones diacrónicas y sincrónicas. Esto implica que la nueva didáctica debe ajustarse a los procedimientos que produzcan aprendizajes significativos, articulando condiciones de organización del orden interno y externo del contexto de aprendizaje, propiciando situaciones convenientes de experiencias, a fin de que el alumno organice la construcción de su propio aprendizaje, mediante herramientas convencionales y virtuales, a través de metodologías que movilicen estructuras cognitivas básicas y complejas, que caracterizan al nivel de educación superior.

Palabras clave: Didáctica, implementación, aulas universitarias, aprendizaje significativo. 


\title{
New role of didactics implemented in university classrooms
}

\begin{abstract}
This research aims to describe the role of didactics implemented in university classrooms, and in turn highlight the relevance of the subject as the central nucleus of this process. The method used is the exhaustive review and analysis of the existing literature in relation to the topic addressed. The main findings of this work were: that the role of didactics implemented in university classrooms requires the articulation of cognitive processes to achieve the development of competencies and capacities through systematic and rational classroom experiences, using various technological tools for access to information and to transfer it in a meaningful way to students, both in face-to-face and virtual classes, in diachronic and synchronic situations. This implies that the new didactics must adjust to the procedures that produce meaningful learning, articulating conditions of organization of the internal and external order of the learning context, promoting convenient situations of experiences, so that the student organizes the construction of their own learning, through conventional and virtual tools, through methodologies that mobilize basic and complex cognitive structures, which characterize the higher education level.
\end{abstract}

Keywords: Didactics, implementation, university classrooms, meaningful learning.

Artículo recibido: 03 nov. 2020 Aceptado para publicación: 07 dic. 2020 Correspondencia domingaelsa@gmail.com Conflictos de Interés: Ninguna que declarar 


\section{INTRODUCCIÓN}

La Didáctica, según Corrales, mencionado en Moreno Méndez, es la Teoría de la enseñanza que tiene por objeto el estudio del proceso de enseñanza de una forma integral, a saber: la instrucción, la enseñanza, las condiciones que propician el trabajo activo y creador de los alumnos y su desarrollo intelectual y el aprendizaje entendido como la asimilación, apropiación activa y consciente de los fundamentos de la ciencia y su aplicación práctica (Moreno Méndez, Rodríguez Quintero, Mera Rodríguez, \& Beltrán Vargas, 2007). Lo mencionado por el autor permite dimensionar a la didáctica como una teoría que incluye un conjunto de principios que orienten el quehacer del trabajo docente, y que debería conducir a la segura formación del educando en todas sus dimensiones.

El Papel de la Didáctica es entonces fundamental, en todos los niveles de la educación; no menos importante es la implementación de las herramientas y teorías que se proponen a través de ella, en el nivel de educación superior. Por ello, en este material, se intenta reconocer: ¿Cuál es el nuevo rol de la didáctica en las aulas universitarias? ¿En qué medida se utiliza sus procedimientos y herramientas tecnológicas para que se produzcan verdaderos aprendizajes constructivos, en los entornos actuales?, ¿De qué manera los docentes se encuentran preparados ante este reto ocasionado por la influencia de la pandemia causada por el Covid 19?

La educación superior ha venido recibiendo el impacto de la transformación a partir de la expansión de los conocimientos y saberes, pero de manera imperativa con la implementación de las tecnologías de la información y la comunicación dentro del proceso educativo, se ha generado un cambio dentro de las pedagogías y en la educación a distancia. (Camacho, (2014, pág. 604).

Hoy día constituye todo un reto el tema de ayornarse hacia la adquisición de nuevas herramientas virtuales que coadyuven con el aprendizaje en red; esta modalidad abrupta de enseñanza y aprendizaje, ha generado grandes retos para el docente y el alumnado en general. Otrora, la enseñanza por medo de los entornos virtuales, constituía una opción, hoy constituye todo un desafío y exigencia en la educación superior.

Vale remarcar, en este sentido, que la didáctica a ser implementada en las aulas universitarias, a partir de esta coyuntura, deberá ser diacrónica, y sincrónica a la vez, ya que requiere de un ajuste constante y pertinente para producir efectos motivadores en los estudiantes, y así responder a la Matética, la cual significa "a quien va dirigido el 
aprendizaje", cuyo sujeto se halla condicionado por el conjunto de situaciones contextuales e históricos (Bruera, 1996). Este proceso diacronizador, implica una actualización constante en los conocimientos y teorías específicas de la disciplina, así como en los procedimientos y estrategias implementados como medio de aprendizaje.

En este trabajo se pretende demostrar que la didáctica universitaria, necesita comprometerse con el desarrollo de los procesos cognitivos de los estudiantes de este nivel de educación. Ante esta perspectiva, es pertinente desarrollar habilidades cognitivas básicas, tales como la lectura, la escritura, la comprensión verbal, numérica, visualización espacial, retención de imágenes, palabras y razonamiento; ya que, según Pinzas, la didáctica universitaria eficaz, se logra a través de un conjunto de procesos que permiten al educando "recibir, interpretar, almacenar, y utilizar la información que les brinda el docente" (Castillo Arredondo \& Cabrerizo Diago, 2005). Tales procedimientos son insoslayables para conectar al estudiante con el conocimiento y su producción; funciones sustantivas de la Enseñanza Universitaria. En este trabajo se describe la importancia de una implementación efectiva de la didáctica en las aulas universitarias, y de qué manera se articulan los procedimientos para que se produzcan los aprendizajes constructivos.

Así mismo, este aprendizaje virtual abre paso a un autoaprendizaje fundamentado en el dominio de la utilización de estos entornos. "La formación con NTICs hace evidente, por su parte, que el aprendizaje no-presencial hace parte del proceso formativo, y su importancia en el mismo. A estos procesos educativos nuevos se los puede denominar “desescolarización” (Chan NÚÑEZ, cit en Grosso, 2004, p.7). En este tiempo es necesario adquirir habilidades y herramientas para el desarrollo de las clases, porque esta exigencia abrupta, no constituye una opción, sino una realidad vivenciada en todos los sistemas educativos de todo el mundo, y en todos los niveles. Este autoaprendizaje es un desafío, tanto para docentes como para los alumnos.

\section{ESTRATEGIAS METODOLÓGICAS O MATERIALES Y MÉTODOS}

El método utilizado para la investigación fue principalmente una revisión y análisis de la literatura relacionada con la didáctica implementada en las aulas universitarias, y su innovación para responder a la situación emergente; el procedimiento consistió en una selección cuidadosa de la literatura referente al tema y la posterior discusión con las autoras del trabajo; y así, llegar a las conclusiones más significativas de este escrito. 


\section{RESULTADOS Y DISCUSIÓN}

\section{Una aproximación al concepto de la didáctica}

La didáctica es una disciplina científico-pedagógica, que tiene como objeto de estudio los procesos y elementos que interactúan para la producción de los aprendizajes esperados. Se trata del área de la pedagogía que se encarga de los sistemas y de los métodos prácticos de enseñanza destinados a plasmar las pautas de las teorías pedagógicas, considerando que las mismas permiten variar el concepto de aprendizaje (Monereo, 2007).

La didáctica no tiene razón de ser si no existe aprendizaje, para los conductistas, el aprendizaje es el cambio relativamente permanente en la conducta de la persona, como respuesta a una situación específica que sería el estímulo; por otro lado, la teoría cognitiva, considera que el aprendizaje es fruto de la actividad intelectual, personal que ocurre cuando la persona pone en marcha determinados procesos de carácter voluntario, junto con procesos menos controlados; a más de otras teorías que interactúan eclécticamente entre ambas (Avolio de Cols, 1998).

Esta visión que conjuga con la teoría cognitiva, exige que el profesor evite la adopción de una postura pasiva, aprovechando con creatividad y amplitud los recursos propios de la disciplina y los factores que en ella confluyen. Ello implica, además, un autoaprendizaje para el docente que deberá implementar nuevas herramientas virtuales ante las necesidades de hoy día. Para ello, tendrá que aplicar una visión estratégica con la que pueda regular su acción y así promover en el estudiante el desarrollo de algunas variables que entran en juego en el aprendizaje autónomo y cooperativo, tales como las cognitivas, motivacionales, afectivas y contextuales. (Chacín \& Briceño, 2008).

Por lo expuesto, se reconoce la necesidad de una Didáctica centrada en el sujeto que aprende, lo cual exige enfocar la enseñanza como un proceso de orientación del aprendizaje, donde se creen las condiciones para que los estudiantes se apropien de los conocimientos, no solamente de manera superficial, sino de manera metacognitiva; que desarrollen habilidades; formen valores y adquieran estrategias que les permitan actuar de forma independiente, comprometida y creadora, como protagonista sustancial de su aprendizaje para una actuación emancipadora ante los problemas emergentes de la sociedad. 
En este sentido, este conjunto de actividades debe hallarse integrado por una educación progresista, que no puede, en el nombre de la disciplina y el orden, castrar la dignidad del educando, su capacidad de oponerse ni tampoco se le debe imponer un quietismo negador de su ser. Esto implica que el educador debe aprovechar cualquier oportunidad para manifestar su compromiso con la realización de un mundo mejor, más justo, menos indecente, más sustancialmente democrático (Freire, 1997). De esta manera, se formará un profesional crítico, prospectivo e innovador en el campo laboral adscripto.

El profesor está llamado al crecimiento de su intelecto y a su superación, apoyándose en su capacidad de autocrítica permanente, sobre la base de la reflexión, el espíritu innovador, y la autoexigencia para aprender a aprender.

Desde esta perspectiva, el quehacer en el campo de la didáctica, se plantea el desarrollo de aprendizajes significativos, por descubrimiento y por asimilación, el cambio conceptual, el desarrollo del potencial intelectual mediante el uso de estrategias de aprendizaje y las interacciones con el ambiente; que constituyen parte de los retos que debe asumir la didáctica para emprender un nuevo camino en el diseño de la enseñanza en las aulas universitarias, más aún que constituye todo un reto la implementación de las nuevas tecnologías de la información y comunicación, y el dominio potencial de herramientas virtuales.

\section{La didáctica como ciencia cognitiva en la educación superior}

En este orden de ideas, a la universidad le corresponde, como ente social, facilitar los procesos de enseñanza - aprendizaje de manera organizada y sistemática. La enseñanza representa el aspecto específico de la práctica educativa, la cual supone la institucionalización del quehacer educativo y la sistematización y organización de éste alrededor de los procesos de enseñanza - aprendizaje, que exigen que la capacidad humana permita el desarrollo de procesos cognitivos para conocer, comprender y actuar sobre el mundo que la rodea para relacionarse con este (Franco Ligarreto, 2017).

Asimismo, Freire propone que la tecnología didáctica aplicada a la educación apuntale al desarrollo de la conciencia socialista en la vida de los individuos, en su interacción estrecha con su entorno social históricamente cambiante (Mészáros, 2008). Sin embargo, la realidad de la Didáctica en las aulas universitarias no constituye hoy, precisamente, este ambiente libre, creativo, donde el estudiante se halle dotado de la autonomía para expresar su sentir, su pensar acerca de las realidades, ya sea por temor a represalias o por 
dudar de su postura ante el hecho discutido; en ese aspecto, lejos aún estamos de dotar un ambiente de discusión genuino sobre los problemas sociales y políticos que hoy requieren atención.

A partir de esta descripción se puede deducir que el impacto que ocasiona la implementación de esta didáctica en las aulas traerá aparejado un egresado estigmatizado por las ideas de sus docentes, que ejercen una influencia dominante sobre las ideas de los jóvenes; por tanto, el profesional egresado se halla siempre supeditado al ejercicio del poder del jefe, o de la autoridad inmediata superior, privándose, de esa manera, de un pensamiento liberador y auténtico a la hora de la toma de decisiones.

La tecnología didáctica es un saber y hacer sistemático en torno al proceso de enseñanzaaprendizaje, buscando su mejor comprensión, aplicación rigurosa y optimización. En este sentido, el sujeto crece y dinamiza sus capacidades, habilidades y conocimientos, dentro de una lógica de incorporación y transferencia en los procesos de operacionalización para la resolución de los problemas emergentes en el contexto, que es la finalidad de la implementación efectiva de la didáctica en las aulas de la educación superior. Para la concreción de dicha comunicación didáctica efectiva, se requiere la interacción significativa entre docentes y estudiantes, creando un clima propicio, que genere un ecosistema receptivo de las vivencias y experiencias que facilite el logro de la comunicación eficaz, que constituye la base de las actuaciones y de las innovaciones que se llevan a cabo en el aula. (Domínguez Garrido, Medina Rivilla, \& Sánchez Romero, 2011)

La didáctica, considerada como ciencia cognitiva plantea los siguientes aspectos integrados para efectivizar la práctica educativa, según Bruera (2)

La didáctica, considerada como ciencia cognitiva, plantea los siguientes aspectos integrados para efectivizar la práctica educativa, según Bruera (2)

Tabla I. Fases de la implementación didáctica.

\begin{tabular}{c|l}
\hline Fases & \multicolumn{2}{|c}{ Permiten: } \\
\hline Reflexión epistemológica & definir el objeto de la enseñanza, delimitar los \\
& campos disciplinarios, $\quad$ seleccionar los \\
& conocimientos, clarificar los métodos y procesos \\
& propios del saber
\end{tabular}




\begin{tabular}{|c|c|}
\hline Variable psicológica & contextualizar el proceso de aprendizaje: nivel de \\
\hline $\begin{array}{l}\text { Estructuración de los } \\
\text { contenidos }\end{array}$ & $\begin{array}{l}\text { establecer una secuencia lógica de los contenidos a } \\
\text { ser desarrollados }\end{array}$ \\
\hline $\begin{array}{l}\text { Especificación de las } \\
\text { tareas }\end{array}$ & $\begin{array}{l}\text { seleccionar las experiencias de aprendizaje en } \\
\text { coherencia con el alcance y la complejidad de las }\end{array}$ \\
\hline Asignación del tiempo & $\begin{array}{l}\text { capacidades a ser logradas. } \\
\text { estimar la duración de las tareas según la } \\
\text { complejidad de los contenidos abordados. }\end{array}$ \\
\hline $\begin{array}{l}\text { Delimitación } \\
\text { competencias }\end{array}$ & $\begin{array}{l}\text { la especificación de los aprendizajes previstos en } \\
\text { cada etapa de enseñanza }\end{array}$ \\
\hline
\end{tabular}

Cuadro adaptado de Ricardo Bruera, por las autoras del artículo.

\section{El estudiante como referente nuclear del diseño curricular universitario}

La Psicología del Aprendizaje universitario adquiere un papel preponderante en el aprendizaje del estudiante, ya que ésta deberá conjugar los procesos cognitivos, la arquitectura y el sistema operativo de la mente del estudiante a la hora de desarrollar sus estudios; por ello, es fundamental fomentar la metacognición.

Este concepto lo han desarrollado distintos teóricos, entre los que se destaca la propuesta de Flavell, quien considera que la metacognición es la "cognición sobre la cognición", es decir, se refiere al conocimiento que uno tiene acerca de los propios procesos y productos cognitivos, lo que significa que cada estudiante puede reflexionar acerca del modo en que incorpora la información y a la vez desarrolla habilidades que les permite emplear asertivamente dichos conocimientos (Flavell, 1993). Se trata, pues, de un proceso mental complejo que requiere de procesos más básicos, aunque incluye otros elementos inherentes a la aprehensión de los conocimientos, tales como estrategias, reglas y heurísticos.

Así mismo, como lo plantean Piaget, J. y Vigotsky, L; es importante considerar las etapas del desarrollo cognitivo del sujeto que aprende, como también optimizar su potencial, refiriéndose a la Zona de desarrollo potencial o Zona de Desarrollo Próximo (ZDP), y atendiendo las etapas del desarrollo del sujeto, sin descuidar ninguna de ellas, aunque se trate del nivel de enseñanza universitaria. Si se considera estas teorías en las aulas universitarias, se obtendrá, efectivamente, el logro de las competencias y capacidades. (Díaz-Barriga y Hernández, 2002). 
Además de esto, se debe tener en cuenta los procesos de socialización y subjetivación de los estudiantes de este nivel; en el de socialización, el mismo incorpora el conjunto de valores y cultura de la sociedad en la que se halla inmerso; por otro lado, el proceso de subjetivación lo conducirá hacia una construcción formativa que deriva de la experiencia social y educativa, para incorporarse asertivamente a su contexto inmediato, desde su modo de ser, de estar y de apropiarse de su mundo circundante (Abdala, Barros, Ceconello, Gunset, Reinaga y Yapur, 2012). Esta es la razón por la cual este proceso de enseñanza en el nivel universitario demande por parte del docente, no solamente los conocimientos específicos de la disciplina que enseña, sino también una vasta preparación acerca de las metodologías y estrategias para su aplicación oportuna en situaciones de aprendizaje (Montes de Oca, Machado Ramírez, 2011).

Esta didáctica centrada en el sujeto que aprende, exige enfocar la enseñanza como un proceso de orientación del aprendizaje, donde se creen las condiciones para que los estudiantes no sólo se apropien de los conocimientos, sino que desarrollen habilidades, formen valores y adquieran estrategias que les permitan actuar de forma independiente, comprometida y creadora, para resolver los problemas emergentes.

Todo ello conlleva la utilización de estrategias y métodos que propicien un aprendizaje intencional, reflexivo, consciente y autorregulado, regido por objetivos y metas propios, como resultado del vínculo entre lo afectivo y lo cognitivo, y de las interacciones sociales y la comunicación, que consideren la diversidad del estudiantado y las características de la generación presente en las aulas universitarias, con la irrupción de las Tecnologías de la Información y las Comunicaciones.

\section{CONCLUSIÓN O CONSIDERACIONES FINALES}

En el presente trabajo se ha puesto el énfasis sobre la consideración de la didáctica como una disciplina pedagógica y de fuerte carácter aplicativo, que puede guiar la actividad del docente universitario, y así, ayudarlo a ser verdaderamente estratégico, a fin de estimular el aprendizaje autónomo en los estudiantes, más aún en circunstancias como las actuales, que impiden el relacionamiento directo entre docente y alumno.

El papel de la didáctica implementada en las aulas universitarias, debe ser enriquecedor para una producción efectiva de los conocimientos deseados, por eso es fundamental integrar el saber didáctico con el saber hacer didáctico; organizando la tarea práctica de manera coherente, que permita al estudiante el desarrollo de competencias y capacidades 
a través de experiencias de aulas, sistemáticas y racionales; mediante instrumentos y metodologías que movilicen estructuras cognitivas básicas y complejas, características de este nivel de educación superior.

Las demandas actuales para este nivel requieren nuevas maneras de acceso al conocimiento y a la información que trascienden la clásica interacción docente-alumnos; requiere pues, además de ello, el dominio de las herramientas digitales que permitan el acceso a entornos virtuales de diversa índole, que coadyuven con un verdadero compromiso por parte del estudiante universitario, para la aprehensión y transferencia de los nuevos conocimientos.

La nueva concepción de la didáctica debe ajustarse a los procedimientos que produzcan aprendizajes significativos, articulando condiciones de organización del orden externo del contexto de aprendizaje; propiciando situaciones convenientes de experiencias a fin de que el alumno organice la construcción de su propio aprendizaje.

Estas reflexiones permiten confirmar, que el perfil del docente universitario demanda la articulación de los conocimientos específicos de la disciplina que enseña, así como también, una vasta preparación en cuanto a las metodologías y estrategias para una aplicación oportuna en situaciones de aprendizaje, tanto en escenarios presenciales y virtuales.

\section{LISTA DE REFERENCIAS}

Abdala, C. Barros, Ma. E., Ceconello, M, Gunset V., Reinaga, D y Yapur, Ma. C., 2012. La Universidad que vivimos. Tucumán: Departamento de publicaciones de la Facultad de Filosofía y Letras de la UNT.

Avolio de Cols, S. (1998). Los proyectos para el trabajo en el Aula. Interrogantes básicos: ¿Por qué?, ¿Para qué?, ¿Qué? y ¿Cómo?. Buenos Aires: Marymar.

Bruera, R. (1996). La Didáctica como Ciencia Cognitiva. Rosario: : CEDIE. .

Camacho, L. J. ((2014). NUEVOS ROLES DE LOS DOCENTES EN LA EDUCACIÓN SUPERIOR: HACIA UN NUEVO PERFIL Y MODELO DE COMPETENCIAS CON INTEGRACIÓN DE LAS TIC. Ciencia y Sociedad, 39(4), 601-640.

Castillo Arredondo, S., \& Cabrerizo Diago, J. (2005). Formación del profesorado en educación superior. Madrid: McGraw-Hill Interamericana. 
Chacín, M., \& Briceño, M. (2008). El profesor universitario y la integración de la didáctica en la enseñanza universitaria. Rev. Paradigma., 29(1).

Díaz-Barriga., F. y Hernández R., G. (2002). Estrategias docentes para un aprendizaje significativo. $2^{\mathrm{a}}$ ed. México: McGraw Hill

Domínguez Garrido, M. C., Medina Rivilla, A., \& Sánchez Romero, C. (2011). La Innovación en el aula: referente para el diseño y desarrollo curricular. Perspectiva educacional, formación de profesores, 61-86.

Flavell, J. H. (1993). El desarrollo cognitivo. Madrid: Visor.

Freire, P. (1997). Pedagogía de la Indignación: Cartas pedagógicas en un mundo revuelto. Del archivo de Ana María Araujo Freire. Buenos Aires: siglo XXI, editores.

Franco Ligarreto, B. C. (2017). La didáctica de las ciencias: un camino para el desarrollo cognitivo. Magisterio.com.

Gómez Sánchez, D., Oviedo Marín, R., \& Martínez López, E. Í. (2011). Factores que influyen en el rendimiento académico del estudiante universitario. Educación y Humanidades, 90-97.

Grosso, J. L. ( septiembre-diciembre de 2004). NTICS, educación virtual, lógicas de la comunicación social y "sociedad del conocimiento":anotaciones sobre el contexto y la responsabilidad social de la Educación Superior a Distancia en ALC. Revista Diálogo Educacional, 4(13), 1-10.

Marulanda Galvis, J. C., Ortiz Botero, E., Moratto Vásquez, N., \& Arcila Rojas, A. P. (2010). Caracterización de egresados de la Universidad CES en las cohortes de 2003, 2005, 2007 y momento “0”. Revista CES Psicología, 50-63.

Mészáros, I. (2008). La Educación más allá del capital. Sociología y Política. Serie pensar desde el sur. Buenos Aires: Siglo XXI editores

Monereo, C. ( 2007). Hacia un nuevo paradigma del aprendizaje estratégico: el papel de la mediación social, del self y de las emociones. Revista electrónica de investigación en psicología de la educació, 5(3), 497-534. 
Montes de Oca Recio N., Machado Ramírez, E.F. (2011). Estrategias docentes y métodos de enseñanza-aprendizaje en la Educación Superior. Revista Humanidades Médicas. Vol. 11, Núm. 3 Cuba.Rec. 20/03/2017 de la pág: http://www.humanidadesmedicas.sld.cu/index.php/hm/article/view/127/81.

Moreno Méndez, J., Rodríguez Quintero, G., Mera Rodríguez, E., \& Beltrán Vargas, L. (2007). Estrategias Didácticas Desarrolladas por los Docentes para Orientar el Trabajo Integrador en la Facultad de Psicología. Psychologia. Avances de la disciplina., 1(1), 133-162,. 